Saudi Journal of Biomedical Research

Abbreviated Key Title: Saudi J Biomed Res ISSN 2518-3214 (Print) IISSN 2518-3222 (Online)

Scholars Middle East Publishers, Dubai, United Arab Emirates Journal homepage: https://saudijournals.com/sjbr

\title{
Kikuchi Fujimoto's Disease- A Case Series with Review of Literature
}

\author{
Dr. Sruthi $\mathrm{S}^{*}$
}

2nd Yr Pathology Postgraduate, Sree Balaji Medical College and Hospitals, 7 Works, Road, Chromepet, Chennai, Tamil Nadu 600044, India

DOI: $10.36348 /$ sjbr.2020.v05i11.002 | Received: 22.10.2020 | Accepted: 01.11 .2020 | Published: 06.11 .2020

*Corresponding author: Dr. Sruthi S

\section{Abstract}

Generalized lymphadenopathy is a common cause of concern for both patients and clinicians. Causes for kikuchi fujimoto's are infections and malignancies. Kikuchi Fujimoto disease (KFD) presents with fever, lymphadenopathy it also presents with systemic involvement. We report the cases of a 5 patients who presented with generalized lymphadenopathy, high fever, skin vasculitis and polyserositis. The lymph-node biopsy shows histiocytotic necrotizing lymphadenitis, suggestive of Kikuchi's disease.

Keywords: Kikuchi's disease, Necrotising histiocytic lymphadenitis.

Copyright $\odot 2020$ The Author(s): This is an open-access article distributed under the terms of the Creative Commons Attribution 4.0 International License (CC BY-NC 4.0) which permits unrestricted use, distribution, and reproduction in any medium for non-commercial use provided the original author and source are credited.

\section{INTRODUCTION}

Kikuchi-Fujimoto disease (KFD), also known as histiocytotic necrotising lymphadenitis, is a rare, benign, presumably underdiagnosed disease, usually of good prognosis. Although initially considered to affect mostly East-Asian women, it has been meanwhile described in all ethnic groups. While the pathogenesis is not clearly understood, the disease probably occurs consequent to immune activation against an infectious agent. It presents with fever accompanied by lymphadenopathy. The laboratory studies resembles other conditions like tuberculosis, lymphoma, sarcoidosis, systemic lupus erythematosus (SLE) and HIV $[1,2]$.

These cases aims to raise awareness for the disease and the importance of including KFD in the differential diagnosis of lymphadenopathy to prevent inappropriate treatments [3].

\section{HISTOLOGICAL FINDINGS}

A 2010 study by Kim et al., [4] noted the following in cutaneous Kikuchi disease patients:

- Slight predominance of CD8+ lymphocytes,

- Lymphohistiocytic infiltration and non neutrophilic karyorrhexis (usually).

\section{CASE SERIES}

\section{Case No 1}

A 21 year old presented with swelling over the lateral aspect of right side of neck x 1 month. Initially was small in size then progressed in size to attain the current size. $\mathrm{C} / \mathrm{O}$ fever for 10 days associated with evening rise of temperature -High grade, associated with chills and rigor.

FNAC - Features suggestive of TB lymphadentis

And the patient was treated for the same, excision biopsy of the nodes was done to arrive at a definitive diagnosis.

\section{Case 2}

A 11 Year male presented with fever and swelling on the right side of the neck.

Examination: Right sided cervical lymphadenopathy with prominent node of $2 \mathrm{cms}$ at level 5

FNAC: Extensive Necrotising Granulomatous Inflammation

\section{Case 3}

A 14 Yr female presented with history of malise weight loss fever and swelling in the right side of neck

FNAC: Suggestive Of Bacterial Lymphadenitis Treatment was given for the same and excision biopsy was done for definitive diagnosis

\section{Case 4}

A $17 \mathrm{yr}$ old female presented with fever and swelling in the right side of the neck FNAC: TB LYMPHADENITIS 


\section{Case 5}

A 13 Yr old male presented with fever and pain in the left side of the neck.

EXAMINATION: Revealed multiple nodes enlarged in the neck at level

FNAC: Cervical Lymphadenopathy

Possibly TB

\section{Microscopy}

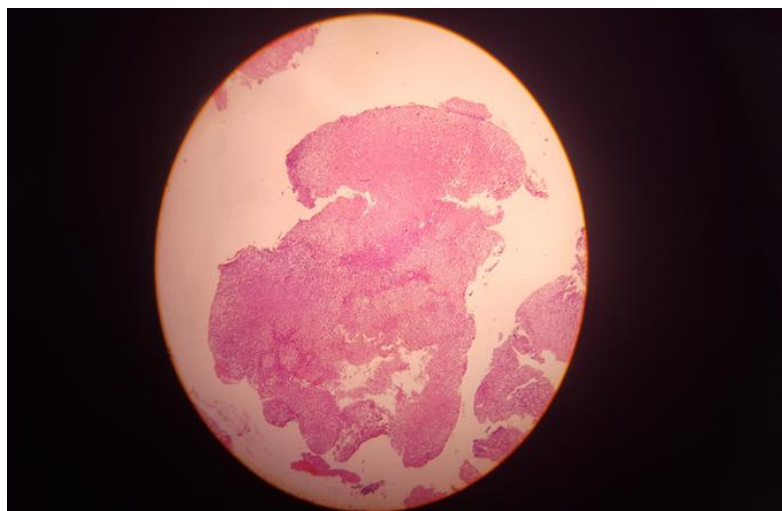

Section show zonal process of the node in the background of follicular hyperplasia

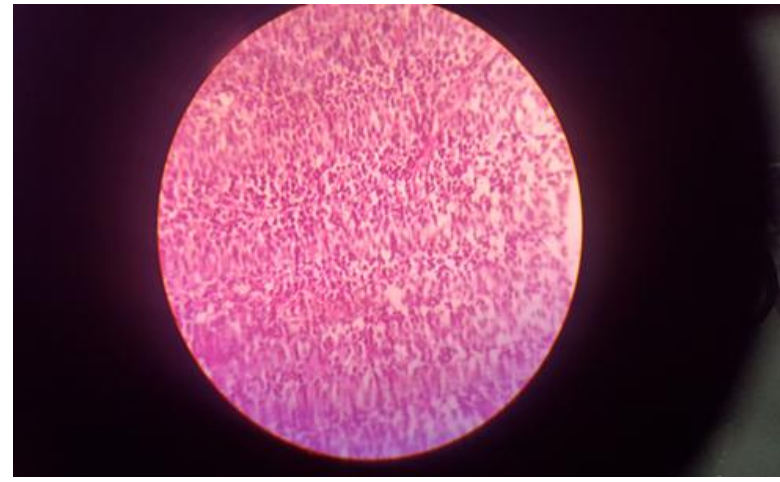

Areas of inflammatory infiltration with areas of necrosis filled with nuclei debri and surrounded by histiocytic proliferation and congested capillaries. Polymorphs and eosinophils are not seen

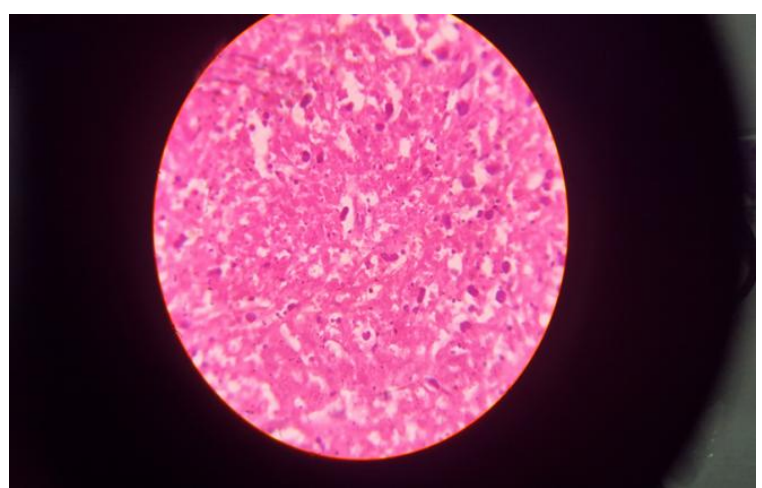

One or more large well defined foci of necrosis containing macrophages with twisted nuclei abundant karryohectic debris and few neutrophils. Immunoblasts and plasmacytoid monocytes are present in periphery of the necrotic zones

\section{DISCUSSION}

Kikuchi-Fujimoto disease is a condition that presents with lymphadenopathy, usually affecting young individuals in their 2 nd and 3 rd decade of life, South east Asian population is usually affected. KFD (Kikuchi fujimoto's disease) is commonly associated with fever and possible symptoms are, myalgia, arthralgia, weight loss, hepatosplenomegaly. The lymphadenopathy most often involves the cervical region, generalised involvement is noted in most cases. On examination moderately enlarged, firm, mobile and nodes can be seen [5]

Our patients presented with a sepsis-like clinical syndrome unresponsive to antibiotic treatment. $\mathrm{C} / \mathrm{O}$ swelling in the neck associated with pain and FNAC showed $\mathrm{Tb}$ lymphadenitis so patients were treated for the same but did not show much improvement.

The clinical features completely resolve after symptomatic therapy, proving that Kikuchi's disease is usually a benign, self-limiting inflammatory condition. On follow-up, with the patients few months after discharge they reported that all symptoms had completely disappeared.

Laboratory study is inconclusive for this disease. Patients frequently present with increased inflammatory markers (C-reactive protein, erythrocyte sedimentation rate), leukopenia or atypical leukocytes, anaemia of chronic disease and, in case of hepatic involvement, slightly elevated liver enzymes.

The diagnosis of KFD is based on excisional lymph node biopsy. The histological findings are follicular hyperplasia, histiocytic and lymphocytic infiltrates and necrotising foci with a high degree of karyorrhexis and the absence of neutrophils and eosinophils. Immunohistochemical studies shows positivity of CD68+ histiocytes, CD123+ plasmacytoid dendritic cells and CD8+ T cells.

In some cases, patients that had been diagnosed with KFD developed systemic lupus erythematosus within months or years after initial presentation. It has been hypothesized that KFD is an excessive apoptotic answer to an infectious agent. SLE is related to insufficient processing of cell debris upon apoptosis, leading to generation of autoantibodies. And the abundant plasmacytoid dendritic cells in KFD lymph nodes, responsible for the type 1- IFN-signature, establishes a link between Kikuchi's disease and SLE [6].

Conditions characterized by lymphadenopathy and fever should be excluded from tuberculosis, leprosy, toxoplasmosis, sarcoidosis, lymphoma, systemic lupus erythematosus. KFD has sometimes been misdiagnosed as non-Hodgkin's lymphoma, 
tuberculosis or relapsing infections. Patients inappropriate courses of treatments based on misdiagnosis

\section{CONCLUSION}

Although KFD is a self-limiting condition, the clinical features of patients with the final diagnosis of KFD have many provisional diagnosis on presentation. It can be easily mistaken for tuberculosis, lymphoma. Early recognition of the disease is is important to avoid aggressive mode of treatment. Treatment of KFD is supportive.

\section{REFERENCES}

1. Mahajan, T., Merriman, R. C., \& Stone, M. J. (2007, April). Kikuchi-Fujimoto disease (histiocytic necrotizing lymphadenitis): report of a case with other autoimmune manifestations. In Baylor University Medical Center Proceedings (Vol. 20, No. 2, pp. 149-151). Taylor \& Francis.
2. Perry, A. M., \& Choi, S. M. (2018). KikuchiFujimoto disease: a review. Archives of pathology \& laboratory medicine, 142(11), 1341-1346.

3. Kaur, S., Mahajan, R., Jain, N. P., Sood, N., \& Chhabra, S. (2014). Kikuchi's disease-a rare cause of lymphadenopathy and fever. J Assoc Physicians India, 62(1), 54-7.

4. Kim, C. H. (1997). U.S. Patent No. 5,694,340. Washington, DC: U.S. Patent and Trademark Office.

5. Lin, H. C., Su, C. Y., Huang, C. C., Hwang, C. F., \& Chien, C. Y. (2003). Kikuchi's disease: a review and analysis of 61 cases. Otolaryngology-Head and Neck Surgery, 128(5), 650-653.

6. Kuo, T. T. (1995). Kikuchi's disease (histiocytic necrotizing lymphadenitis). A clinicopathologic study of 79 cases with an analysis of histologic subtypes, immunohistology, and DNA ploidy. The American journal of surgical pathology, 19(7), 798-809. 